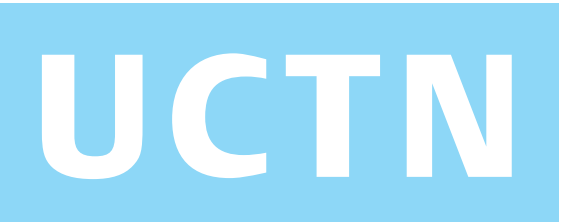

\title{
Endoscopic mucosal resection of a gastric bronchogenic cyst that was mimicking a solid tumor
}

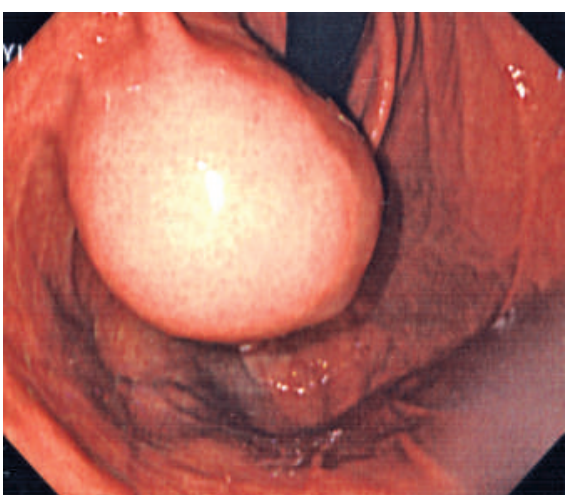

Figure 1 Endoscopy revealed a large, rounded, nearly pedunculated mass, with normal overlying mucosa, in the cardia.

Gastric bronchogenic cysts are extremely rare congenital cystic lesions that originate from the ventral foregut during the 3 rd to 7 th weeks of embryonic development [1]. These lesions can be identified by computed tomography (CT) and magnetic resonance imaging but they are quite often misclassifed as solid tumors [2]. Although endoscopic ultrasound (EUS) is the most valuable diagnostic modality for distinguishing between cystic and noncystic lesions, the similarity of an intramurally located bronchogenic cyst to a smooth muscle tumor or gastrointestinal stromal tumor on EUS makes the diagnosis difficult [2]. We describe here a patient with an intramural bronchogenic cyst that mimicked a solid tumor and which was diagnosed and treated by endoscopic mucosal resection (EMR).

A 38-year-old woman was referred to us with a gastric submucosal tumor. Initial esophagogastroduodenoscopy demonstrated a nearly pedunculated mass with normal overlying mucosa in the cardia (Figure 1). This lesion was soft, with a positive cushion sign. EUS showed this to be an echo-poor mass lesion, $7 \times 5 \mathrm{~cm}$ in size, arising within the submucosal layer (Figure 2). An abdominal CT scan revealed a solid mass originating from the gastric wall (Figure 3). The presumptive diagnosis, based on EUS and CT, was gastrointestinal stromal tumor. However, some type of developmental or complicated cyst

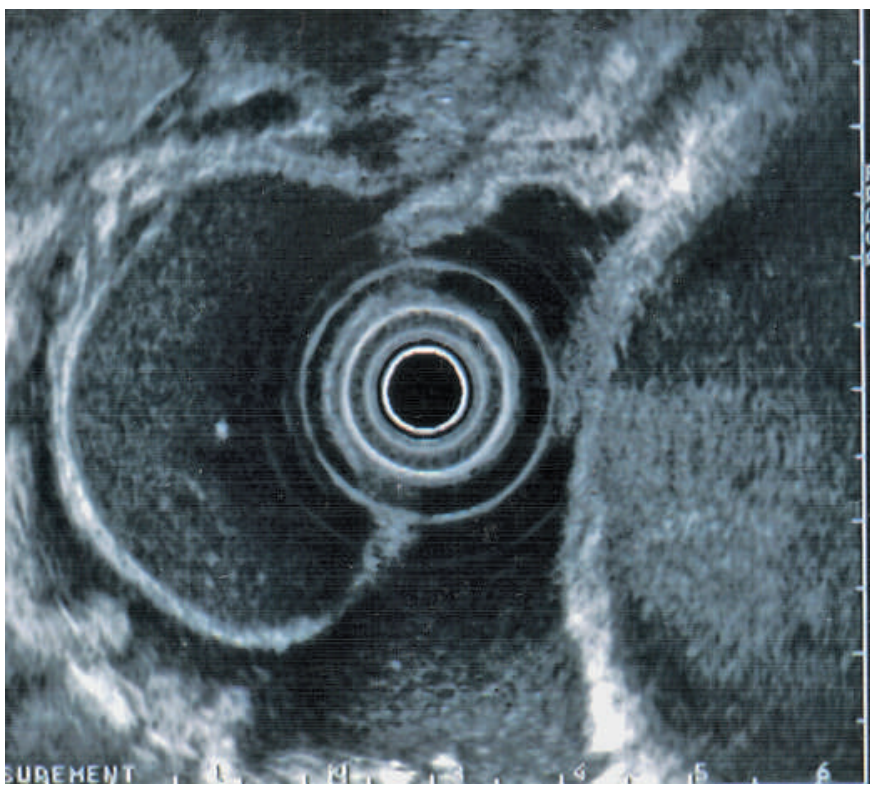

Figure 2 Endoscopic ultrasound image showing a homogeneous, echo-poor mass lesion, $7 \times 5 \mathrm{~cm}$ in size, arising within the submucosal layer.

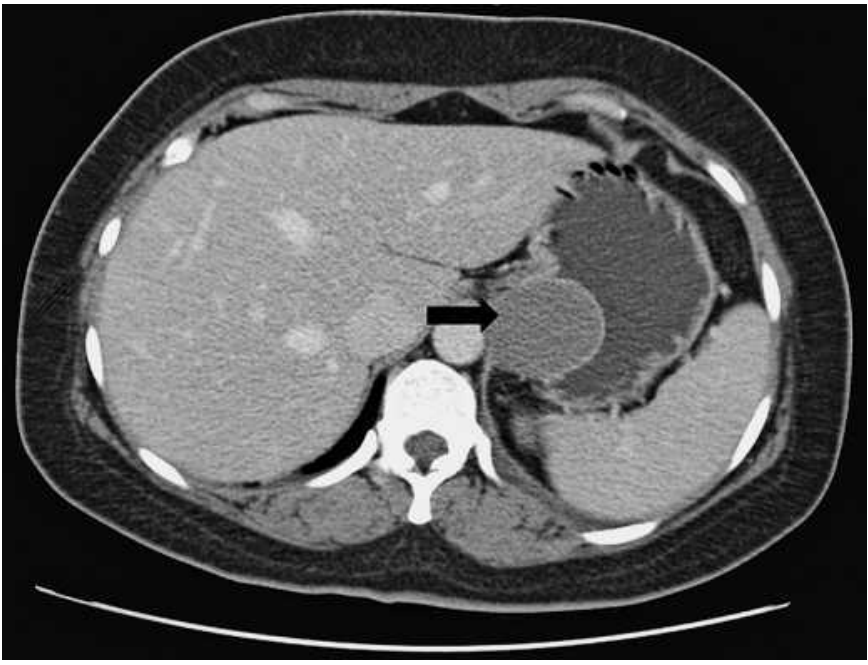

Figure 3 Abdominal computed tomography showed a solid mass (arrow) originating from the gastric wall.

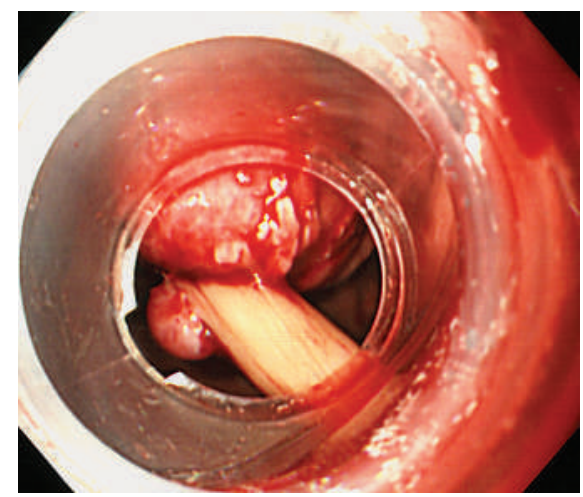

Figure 4 Serous yellow fluid gushed out after the upper portion of the lesion was resected with a transparent cap and a snare. containing dense material was also a possibility because of the positive cushion sign. We decided to perform an endoscopic deroofing in order to obtain a definite diagnosis and to plan treatment. After the upper portion of the lesion was resected, serous yellow fluid gushed out (Video 1, Figure 4). Histological examination revealed that the wall of the resected cyst was composed of ciliated, pseudostratified epithelium, which was similar to that found in bronchogenic cysts (Fig-

DOI: $10.1055 / s-2006-944862$ 

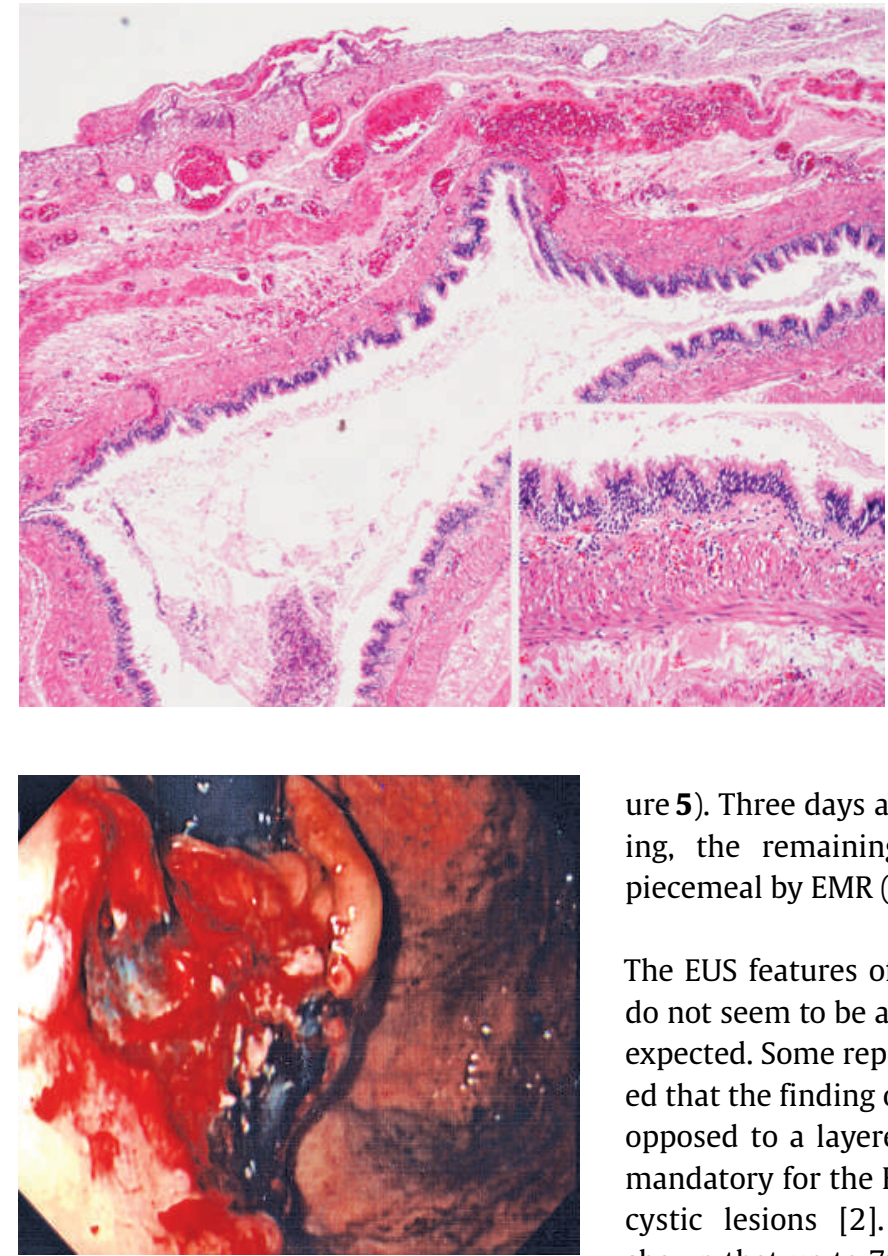

Figure 6 The remaining cyst was resected completely by piecemeal endoscopic mucosal resection.

\section{Video 1}

The mucosa covering the lesion was incised with a snare and the upper portion of the lesion was resected with a transparent cap and a snare. Serous yellow fluid gushed out from the lesion.

\section{Video 2}

Three days after the initial deroofing, the remaining cyst was resected piecemeal by endoscopic mucosal resection, using a transparent cap after submucosal injection. Endoscopy was performed 2 months later, and no obvious residual remnant of the cyst was seen.
Figure 5 Histological views showing the wall of the resected cyst to be composed of ciliated, pseudostratified epithelium (hematoxylin \& eosin, original magnification $\times 40$ (a) and $\times 200(\mathbf{b}))$.

ure 5). Three days after the initial deroofing, the remaining cyst was resected piecemeal by EMR (Video 2, Figure 6).

The EUS features of developmental cysts do not seem to be as uniform as might be expected. Some reports have demonstrated that the finding of an echoic pattern as opposed to a layered-wall pattern is not mandatory for the EUS diagnosis of these cystic lesions [2]. Other reports have shown that up to $70 \%$ of such lesions are misdiagnosed as solid mass lesions on CT and magnetic resonance imaging [3]. This difficulty may be attributed to the very thick proteinaceous contents of these cysts [4]. Although the choice of treatment of intramural bronchogenic cysts remains controversial, EMR could be a good alternative [5]. When a lesion is suspected to be a solid tumor on the basis of EUS and CT investigations, and has a positive cushion sign, the differential diagnosis of a developmental cyst should be considered, and EMR could be used for curative treatment.

Endoscopy_UCTN_Code_CCL_1AB_2AD_3AB

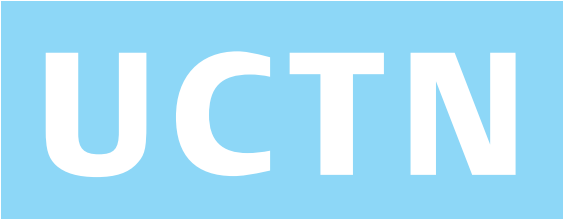

S.-H. Lee', D. H. Park ${ }^{1}$, J. H. Park' ${ }^{1}$, H.-S. Kim1', S.-H. Park', S.-J. Kim', M.-H. Oh ${ }^{2}$

${ }^{1}$ Division of Gastroenterology and Hepatology, Department of Internal Medicine, Soon Chun Hyang University College of Medicine, Cheonan Hospital, Cheonan, Korea

${ }^{2}$ Department of Pathology, Soon Chun Hyang University College of Medicine, Cheonan Hospital, Cheonan, Korea.

\section{References}

${ }^{1}$ Rubio CA, Orrego A, Willen R. Bronchogenic gastric cyst: a case report. In Vivo 2005; 19 : $383-385$

2 Westerterp M, van den Berg JG, van Lanschot $\mathrm{JJ}$ et al. Intramural bronchogenic cysts mimicking solid tumors. Endoscopy 2004; 36: 1119- 1122

${ }^{3}$ Fazel A, Moezardalan K, Varadarajulu S et al. The utility and the safety of EUS-guided FNA in the evaluation of duplication cysts. Gastrointest Endosc 2005; 62: 575-580

${ }^{4}$ Wiech T, Walch A, Werner M. Histopathological classification of nonneoplastic and neoplastic gastrointestinal submucosal lesions. Endoscopy 2005; 37: 630-634

${ }^{5}$ Sashiyama H, Miyazaki S, Okazaki Y et al. Esophageal bronchogenic cyst successfully excised by endoscopic mucosal resection. Gastrointest Endosc 2002; 56: 141 - 145

\section{Corresponding Author}

\section{S.-H. Lee, M.D.}

Division of Gastroenterology,

Department of Internal Medicine Soonchunhyang University Hospital 23-20 Bongmyung-dong

Cheonan city

Choongnam 330-100

South Korea

Fax: $\quad$ +82-41-574-5762

E-mail: ygun99@hanmail.net 